\title{
WIGNER-RACAH DESCRIPTION OF THE FREE-ELECTRON MODEL *
}

\author{
T. LULEK \\ A. Mickiewicz University, Institute of Physics, \\ Matejki 48/49, 60-769 Poznań, Poland \\ (Received July 15, 1991; in final form September 6, 1991)
}

\begin{abstract}
A version of Wigner-Racah type of approach is proposed for the model of a free-electron gas in a cubic box. The approach bases on the structure of fibre bundle in description of states of a single electron. Contrary to the tight binding model, the base of the bundle is the reciprocal space rather than the positional one. An elementary quantity of the approach is the star of quasi-momentum. Such a treatment reveals the degeneracy of energy levels of the system of $N$ electrons, in particular of the ground Fermi level, for an uncomplete filling of stars. The associated classification of errergy levels and corresponding states can be performed within an atomic-like Wigner-Racah scheme.
\end{abstract}

PACS numbers: $02.20 .+b, 71.45 .-\mathrm{d}, 75.10 . \mathrm{Lp}, 75.20 .-\mathrm{g}$

\section{Introduction}

In the present paper we are going to discuss some multiparticle aspects of the model of a free electron in a box along to the Wigner-Racah approach for multielectron states in atoms [1-3]. We aim to point out that this case can be - in principle - described in a way similar to the tight binding model [4-8], where the fibre structure of space of crystalline LCAO spinorbitals carries all essential information on geometric selection rules, whereas the factorization of this space into positional and spin parts allows one to construct symmetry adapted multielectron states in various coupling schemes. It yields discussion of statistics $[5,7,8]$, bilinear invariants [6], Cooper pairs [7], and other features of electron correlations [8].

*The work has been supported by the Ministry of Education research project $\mathrm{P} / 04 / 250 / 90-2 / \mathrm{V} / 1-9$. 
Single-electron spaces of both tight binding and free-electron models admit a fibre structure and allow for appropriate factorizations. The main difference between these models is that the base of the single-electron bundle for the tight binding model is the set of centres, i.e. a subset of the "real space", whereas that for the free-electron case is the discrete Brillouin zone, which is a subset of the reciprocal space of a crystal.

An intriguing feature of such a Wigner-Racah type of approach to the free-electron model is degeneracy of multiparticle energy levels. In particular, the Fermi level, i.e. the ground level-for this model, is usually assumed tacitly as non-degenerate. Actually, it exhibits - in general - a high degeneracy. We aim here to point out the corresponding degrees of freedom for possible constructions of various correlated states belonging to the ground level of the system of $N$ non-interacting electrons.

\section{Basic model assumptions}

We consider the system of $N$ non-interacting electrons, confined to a box. Let the macroscopic geometric symmetry of the box be given by a point group $Q$. Hereafter we assume that the box is a cube of the edge $Q a$, so that $a$ corresponds to the lattice constant when compared to microscopic models. Then $Q$ is the octahedral group $O_{h}$.

The space $L$ of single-electron states of this model is spanned on orthonormal basis states $\mid k \sigma)$, where $k=(2 \pi / Q a)\left(n_{1}, n_{2}, n_{3}\right)$ is the wave vector of quasi-momentum, with integers

$$
n_{i}=0, \pm 1, \pm 2, \cdots, \quad i=1,2,3
$$

defined by standard Born-Kármán periodic quantization conditions, and $\sigma= \pm 1 / 2$ is the spin variable for a single electron. The corresponding energy levels form a ladder given by

$$
\mathcal{E}_{\nu}=\mathcal{E}_{0} \nu
$$

where

$$
\mathcal{E}_{0}=\frac{2 \pi^{2} \hbar^{2}}{m Q^{2} a^{2}}
$$

is the elementary energy distance, and

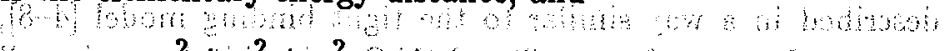

$$
\begin{aligned}
& \nu=n_{1}^{2}+n_{2}^{2}+n_{3}^{2}
\end{aligned}
$$$$
\text { montongth }
$$

is a non-negative integer resulting from the quantization (1) and labelling the rungs of the ladder of energy levels.

The degeneracy $d(\nu)$ of the $\nu$-th rung is the doubled (for reason of spin) number of integer solutions of the diophantine equation (4) for triads $\left(n_{1}, n_{2}, n_{3}\right)$. If a wave vector $k$ is such a solution, then the set

$$
\gamma \equiv \gamma[k]=\{q k \mid q \in Q\}
$$


yields also solutions with the same $\nu$. This set constitutes the star of the wave vector $k$, i.e, the orbit generated by the geometric symmetry group $Q$ from the initial $k$. This orbit is characterized by the stability group

$$
\boldsymbol{H}=\{q \in \boldsymbol{Q} \mid q k=k\}
$$

of the element $k$, or - more accurately - by the class

$$
[H]=\left\{q H q^{-1} \mid q \in Q\right\}
$$

of subgroups, conjugated with $\boldsymbol{H}$ in $Q$, called the epikernel (cf. [9] and references therein for detail) The rung $\nu$ corresponds to the collection of all stars with the same length $|k|$ :

Let us consider now the action $T$ of the group $Q$ on the set $\beta$ of all wave vectors admissible by quantization (1). Evidently, each subset $\beta(\nu)$ of wave vectors with energy $\mathcal{E}_{\nu}$ is invariant under this action. Let

$$
\mathcal{T}_{\nu} \approx \sum_{\eta \in i(Q)} m(\nu, \eta) R_{\eta}
$$

be the stratification of the set $\beta(\nu)$, ite the decomposition of restriction $\mathcal{T}_{\nu}$ of the action $\mathcal{T}$ to the set $\beta(\nu)$ into transitive constituents $R_{\eta}$ with multiplicities $m(\nu, \eta)$. The stratification (8) yields the classification scheme for the initial single-electron orthonormal basis states, denoted by

$$
|\nu \eta a k \sigma\rangle
$$

where $\nu=0,1,2, \ldots$ is the rung of energy ladder (2), $\eta \in \tilde{l}(Q), \tilde{l}(Q)$ is the lattice of all distinct epikernels of the group $Q$, and $a=1,2, \ldots, m(\nu, \eta)$ is the repetition index for the star $\gamma \subset \beta(\nu)$ with the epikernel $\eta$. The single-electron degeneracy is

$$
d(\nu)=2 \sum_{\eta \in \hat{\imath}(Q)} m(\nu, \eta)|\gamma|
$$

where $|\gamma|$ denotes the number of arms of the star $\gamma$, i.e. the (linear) dimension of the transitive representation $R_{\eta}$.

The space of quantum states of the system of $N$ non-interacting electrons in a box is constructed by antisymmetrization $L^{\left\{1^{N}\right\}}$ of the $N$-th tensor power $L^{N}$ of the single-electron space $L$. Thus the Fock space of the model is

$$
F=\sum_{N=0}^{\infty} \oplus L\left\{1^{N}\right\}
$$

In particular, $N=0,1$, and 2 corresponds respectively to the electron vacuum space $\left(\operatorname{dim} L\left\{1^{\circ}\right\}=1\right)$, to single-electron space $L\left\{1^{1}\right\}=L(L$ is a Hilbert space spanned on the denumerable orthonormal set (9)), and to space of electron pairs.

The structure of energy levels of the system of $N$ free electrons is completely determined in terms of occupation numbers of single-electron levels (9), consistent with the Pauli principle In the case of the ground state of the system, the 
single-electron levels are occupied consecutively according to increase of energy. Each rung $\nu$ can be occupied by at most $d(\nu)$ electrons, in an analogy to an atomic $n l$-shell, which encloses $2(2 l+1)$ electrons. Let

$$
N=N_{0}+N^{\prime},
$$

where

$$
N_{0}=\sum_{\nu=0}^{\nu_{\mathrm{F}}-1} d(\nu)
$$

is the number of electrons on completely filled inner rungs, and $N^{\prime}$ - the number of electrons in the outer rung $\nu_{\mathrm{F}}$. Thus $N^{\prime}$ is subjected to the constraints

$$
0<N^{\prime} \leq d\left(\nu_{\mathrm{F}}\right) \text {. }
$$

We refer hereafter to $\nu_{\mathrm{F}}$ as the Fermi rung. The case $N^{\prime}=d\left(\nu_{\mathrm{F}}\right)$ corresponds to complete occupation of the Fermi rung, whereas cases $N^{\prime}<d\left(\nu_{\mathrm{F}}\right)$ imply only partial filling, and thus a degeneracy of the ground level of the $N$-electron system. Clearly, the atomic analogy of the former case is the eighth column of the periodic table of elements, i.e. noble gases with all $n l$ shells either completely filled or empty, whereas the latter corresponds roughly to the rest of this table.

The energy $E(N)$ of the ground level of the system is

$$
E(N)=\sum_{\nu=0}^{\nu_{\mathrm{F}}-1} \mathcal{E}_{0} \nu d(\nu)+\mathcal{E}_{0} \nu_{\mathrm{F}} N^{\prime}
$$

and the degeneracy

$$
D(N)=\left(\begin{array}{c}
d\left(\nu_{\mathrm{F}}\right) \\
N^{\prime}
\end{array}\right)
$$

This degeneracy varies with $N$ rather rapidly. It is $D(N)=1$ for the fully filled Fermi rung $\left(N^{\prime}=d\left(\nu_{\mathrm{F}}\right)\right)$, whereas for the half-filled rung consisting of a single star $\gamma$ it takes on the value

$$
D(|\gamma|)=\left(\begin{array}{c}
2|\gamma| \\
|\gamma|
\end{array}\right)
$$

E.g. for the general star of the octahedral group $Q=O_{h}(|\gamma|=48)$ we have

$$
D=\left(\begin{array}{c}
96 \\
48
\end{array}\right) \sim 10^{28}
$$

the value which, e.g., exceeds the Avogadro number.

The ground level is therefore highly degenerated, and this feature seems to us to be overlooked in standard treatments. In fact, the textbook definition of a ground state in terms of Fermi surface, i.e. essentially in terms of occupation numbers, yields only a mixture, or density matrix, which coincides with a pure quantum state only in cases of totally filled rungs. Other cases admit a large freedom of choice of various mutually orthogonal ground states, with distinct physical 
properties. A natural way of systematic studying of this freedom is suggested by a Wigner-Racah type of approach, starting from a single star $\gamma$.

In this way we meet the problem of classification and construction of an orthonormal basis of $N$-electron spaces $L(\gamma)\left\{1^{N}\right\}$, with $N=0,1,2, \ldots, 2|\gamma|$, starting from a finite-dimensional single-electron space

$$
L(\gamma)=\operatorname{lc}_{\mathcal{C}}\left\{\Psi_{k_{\sigma}} \mid k \in \gamma, \sigma \in \tilde{s} \equiv\{ \pm 1 / 2\}\right\},
$$

with lc $c_{\mathcal{C}}$ denoting the linear closure of the set $\{\ldots\}$ over the field $\mathcal{C}$ of complex numbers. This problem bears an evident resemblance to atomic [10], nuclear [11], and quantum-chemical [12] cases of construction of multiparticle states from a given, finite set for a single particle. In order to give an idea of size of the actual problem we observe that the number $|\gamma|$ of wave vectors in a star $\gamma$ for the group $O_{h}$ varies from 1 (the unit representation) to 48 (the regular one), so that for the maximal occupationnumber $2|\gamma|$ we have

$$
2 \leq 2|\gamma| \leq 96 .
$$

It'should be compared to the quantity $2(2 l+1)$ for the atomic case. Thus, e.g., the tetragonal star $\gamma$, defined by the epikernel $\eta=\left[C_{4 v}\right]$, and consisting of six vectors $\pm k e_{i}, i=1,2,3$ along tetragonal axes, can be occupied by at most 12 electrons. The classification problem for all $N$-electron states, $N=0,1, \ldots, 12$, is in this case intermediate between that for $d$-electrons $(2(2 l+1)=10)$ and $f$-electrons $(14)$. The scale of the problem increases considerably for larger stars.

We depict in the sequel some general aspects of the Wigner-Racah approach to this problem. We stress here our attention on two features. First, the similarity of our problem to the atomic case is deepened by the observation that the single-electron space $L(\gamma)$ can be factorized as

$$
L(\gamma)=L_{0}(\gamma) \otimes L_{s}
$$

into the orbital factor

$$
L_{0}(\gamma)=\operatorname{lc}_{\mathcal{c}} \gamma
$$

and the spin space

$$
L_{s}=\operatorname{lc}_{\mathcal{C}} \tilde{s} .
$$

Equation (21) bears an evident resemblance to the spin-orbit factorization in the atomic case. Second, the orbital space $L_{0}(\gamma)$ of the free-electron case is spanned on a well defined basis $\gamma$. This basis is an orbit of a transitive representation of the group $Q$, and thus the construction of $N$-electron states involves multiple coupling of transitive representations by means of Mackey theorem. 


\section{Factorization and multielectron spaces}

The factorization (21) of the single-electron space $L(\gamma)$ implies the isomorphism

$$
L(\gamma)^{N} \approx L_{0}(\gamma)^{N} \otimes L_{s}^{N}
$$

of the $N$-th tensor power space $L(\gamma)^{N}$ to the direct product of such powers of the orbital and spin factors. Let

$$
\tilde{N}=\{j \mid j=1,2, \ldots, N\}
$$

denote the set of labels of electrons. The set of mappings

$$
\gamma^{\tilde{N}}=\left\{f_{0}: \tilde{N} \rightarrow \gamma\right\}
$$

and

$$
\tilde{s}^{\tilde{N}}=\left\{f_{s}: \tilde{N} \rightarrow \tilde{s}\right\}
$$

i.e. of all orbital

$$
f_{0}=\left|k_{1}, \ldots, k_{N}\right\rangle
$$

and spin

$$
\left.f_{s}=\mid s_{1}, \ldots, s_{N}\right)
$$

configurations of $N$ distinguishable electrons on the star $\gamma$ are bases of factors of the r.h.s. of Eq. (24), i.e.

$$
\begin{aligned}
& L_{0}(\gamma)^{N}=\operatorname{lc}_{\mathcal{C}} \gamma^{\tilde{N}}, \\
& L_{s}^{N}=\operatorname{lc}_{\mathcal{C}} \tilde{s}^{\tilde{N}} .
\end{aligned}
$$

The quantum-mechanical principle of indistinguishability of identical electrons is realised in terms of the Pauli group $\Sigma_{N}$, i.e. the symmetric group on the set $\tilde{N}$, with permutations denoted by

$$
\sigma=\left(\begin{array}{c}
j \\
\sigma(j)
\end{array}\right), \quad j \in \tilde{N}
$$

The action $\mathcal{P}$ of the Pauli group $\Sigma_{N}$ in the space $L(\gamma)^{N}$ can be factorized along Eq. (24) as

$$
\mathcal{P}=\mathcal{P}_{0} \otimes \mathcal{P}_{s},
$$

with the factor actions determined by

$$
\mathcal{P}_{0}(\sigma)=\left(\begin{array}{c}
f_{0} \\
f_{0} \circ \sigma^{-1}
\end{array}\right), \quad f_{0} \in \gamma^{\tilde{N}}, \quad \sigma \in \Sigma_{N}
$$


and

$$
\mathcal{P}_{s}(\sigma)=\left(\begin{array}{c}
f_{s} \\
f_{s} \circ \sigma^{-1}
\end{array}\right), \quad f_{s} \in \tilde{s}^{\tilde{N}}, \quad \sigma \in \Sigma_{N}
$$

In a more explicit notation we have, e.g.

$$
f_{0} \circ \sigma^{-1}=\left|k_{\sigma^{-1}(1)}, \ldots, k_{\sigma^{-1}(N)}\right\rangle,
$$

etc. These actions are linear representations and thus they can be decomposed into irreducible representations $A$ of the Pauli group $\Sigma_{N}$, with each $\Lambda$ being a Young diagram, labelled by a proper partition of $N$, i.e.

$$
\begin{aligned}
& \mathcal{P}_{0}=\sum_{\Lambda} m\left(\mathcal{P}_{0}, \Lambda\right) \Lambda, \\
& \mathcal{P}_{s}=\sum_{\Lambda} m\left(\mathcal{P}_{s}, \Lambda\right) \Lambda,
\end{aligned}
$$

where $m\left(\mathcal{P}_{0}, \Lambda\right)$ and $m\left(\mathcal{P}_{s}, \Lambda\right)$ are the corresponding multiplicities.

Let

$$
\Lambda=\left(\lambda_{1}, \ldots, \lambda_{|A|}\right),
$$

with

$$
\sum_{i=1}^{|\Lambda|} \lambda_{i}=N, \quad \lambda_{i} \geq \lambda_{i+1}>0,
$$

be a proper partition of $N$. A standard representation theory for the symmetric group implies selection rules

$$
\begin{aligned}
& |\Lambda|>|\gamma| \Longrightarrow m\left(\mathcal{P}_{0}, \Lambda\right)=0, \\
& |\Lambda|>2 \Longrightarrow m\left(\mathcal{P}_{s}, \Lambda\right)=0
\end{aligned}
$$

i.e. that non-zero irreducible subspaces of the orbital (spin) space $L_{0}(\gamma)^{N}\left(L_{s}^{N}\right)$ occur only for such Young diagrams $\Lambda$ which have not more than $|\gamma|(2)$ parts.

Selection rules (41)-(42) imply that the only Young diagrams $\Lambda$, admissible in Eqs. (37) and (38), are

$$
\Lambda=\left\{2^{\nu_{2}} 1^{\nu_{1}}\right\}
$$

and

$$
\Lambda^{\operatorname{Tr}}=\left\{\nu_{1}+\nu_{2}, \nu_{2}\right\}
$$

for orbital and spin case, respectively. Here, $\nu_{1}$ and $\nu_{2}$ are non-negative integers satisfying

$$
\nu_{1}+2 \nu_{2}=N \text {. }
$$


They have the meaning of occupation numbers for the single-electron orbital space $L_{0}(\gamma): \nu_{1}\left(\nu_{2}\right)$ denotes the number of singly (doubly) occupied arms of the star $\gamma$. We have also

$$
\nu_{1}=2 S,
$$

where $S$ is the total spin of the system.

The orbital and spin multielectron spaces can be decomposed into subspaces irreducible under the Pauli group $\Sigma_{N}$ as

$$
\begin{aligned}
& L_{0}(\gamma)=\sum_{\Lambda}^{\prime} \sum_{t} \oplus L_{0}^{\Lambda t}(\gamma), \quad t \in \tilde{m}\left(\mathcal{P}_{0}, \Lambda\right) \\
& L_{s}^{N}=\sum_{\Lambda}^{\prime} \sum_{u} \oplus L_{s}^{\Lambda^{T r} u}, \quad u \in \tilde{m}\left(\mathcal{P}_{s}, \Lambda^{\operatorname{Tr}}\right)
\end{aligned}
$$

where the prime denotes the summation over all Young diagrams $\Lambda$, satisfying condition (45), and $\tilde{m}\left(\mathcal{P}_{0}, \Lambda\right)$ and $\tilde{m}\left(\mathcal{P}_{s}, \Lambda\right)$ are sets of repetition labels, imposed by decompositions (37) and (38), respectively. Then, the total space $L^{\left\{1^{N}\right\}}$ has the form

$$
\begin{aligned}
& L^{\left\{1^{N}\right\}}=\sum_{\Lambda}^{\prime} \sum_{t} \sum_{u} \oplus\left(L_{0}^{A t}(\gamma), L_{s}^{A^{\mathrm{Tr}} u}\right)^{\left\{1^{N}\right\}}, t \in \tilde{m}\left(\mathcal{P}_{0}, \Lambda\right), \\
& u \in \tilde{m}\left(\mathcal{P}_{s}, \Lambda^{\mathrm{Tr}}\right)
\end{aligned}
$$

where the symbol $(,)^{\left\{1^{N}\right\}}$ stands for the (unique) antisymmetric one-dimensional subspace of the direct product

$$
L_{0}^{A t}(\gamma) \otimes L_{s}^{\Lambda_{*}^{T t}}
$$

corresponding to mutually transposed Young diagrams (43) and (44).

Equation (49) points out the structure of the space $L\left\{1^{N}\right\}$, imposed by the factorization (24). It is the direct sum

$$
L^{\left\{1^{N}\right\}}=\sum_{\Lambda}^{\prime} \oplus L_{A}^{\left\{1^{N}\right\}}
$$

of subspaces

$$
L_{\Lambda}^{\left\{1^{N}\right\}}=\operatorname{lc} c\left(\tilde{m}\left(\mathcal{P}_{0}, \Lambda\right) \times \tilde{m}\left(\mathcal{P}_{s}, \Lambda^{\operatorname{Tr}}\right)\right)
$$

labelled by Young diagrams $A$, which define permutational properties (or statistics) of the orbital and spin factors. Each such $\Lambda$-subspace is spanned on the cartesian product (51) of repetition labels $t, u$ related to orbital and spin part. The set $\tilde{m}\left(\mathcal{P}_{s}, \Lambda^{\operatorname{Tr}}\right)$ of spin repetition labels $u$ can be readily identified as

$$
\tilde{m}\left(\mathcal{P}_{s}, \Lambda^{\mathrm{Tr}}\right)=\{M=-S,-S+1, \ldots, S\},
$$


i.e. the set of all projections $M$ of the total spin $S$, associated with the Young diagram $\Lambda$ through Eq. (46). Thus the principal problem of classification of multielectron states within a given star $\gamma$ consists in construction of a convenient set $\tilde{m}\left(\mathcal{P}_{0}, \Lambda\right)$ of repetition labels for irreducible representations $\Lambda$ of the Pauli group $\Sigma_{N}$, acting in the orbital space $L_{0}(\gamma)^{N}$. In other words, the problem consists in finding some physically reasonable crystal counter-parts $\Lambda t S$ of atomic Russel-Saunders $L S$ terms.

\section{Discussion}

We have shown that the multielectron states of the free-electron model can be constructed in a way similar to Wigner-Racah approach in the case of atoms. Occupation of single-electron states consistent with the energy ladder (2) implies a shell structure, with $\nu$-th shell consisting of a single or several stars $\gamma$, and enclosing at most $d(\nu)$ electrons. Thus the total number $N$ of electrons can be divided into the diamagnetic part $N_{0}$ corresponding to closed shells, and $0<N^{\prime} \leq d\left(\nu_{\mathrm{F}}\right)$ outer electrons of the Fermi rung $\nu_{\mathrm{F}}$. We observe that the ground level of the system is $D(N)$-tuply degenerated, with $D(N)$ given by Eq. (16). Various states of the ground manifold can thus exhibit different paramagnetic properties.

The factorization (24) of the single-electron space $L$ into the orbital factor $L_{0}(\gamma)$ and spin factor $L_{s}$ yields a classification of states by means of the action of the Pauli group $\Sigma_{N}$, which realises the principle of indistinguishability. Various types of permutation symmetry, i.e. various statistics of orbital states of the $N$-electron system are characterised by Young diagrams $\Lambda$ of the form (43), satisfying the condition (45) (two-electron diagrams). Such a diagram defines, in particular, the total spin $S$ corresponding to given statistic $\Lambda$ (Eq. (46)). The condition

$$
0 \leq 2 S \leq N^{\prime}
$$

defines possible values of the total spin in the ground manifold, and thus provides a review of admissible kinds of spin paramagnetism. The diagram $\Lambda$ admits also various orbital states, with properties specified by appropriate constructions of orthonormal bases in the orbital space $L_{0}(\gamma)^{N}$. In particular, one can construct states with some paramagnetic properties, as crystalline analogies of atomic Hund terms (with maximal orbital angular momentum for the manifold with maximal $\operatorname{spin} S)$.

The problem of convenient classification of orbital states is similar to that for atomic (Wybourne [10]) or molecular case (Karwowski [12] and references therein), and its roots have recourse to the duality of Weyl [14] between the mutually centralising actions of the Pauli symmetric group $\Sigma_{N}$ and the unitary group $U_{|\gamma|}$ in the tensor-power orbital space $L_{0}(\gamma)^{N}$. E.g. the classification problem for atomic $p^{N}$ configuration is exhausted by $L S$ quantum numbers, the case $d^{N}$ requires extra the seniority number $v$, and the case $f^{N}$ is partially resolved by additional classification numbers $W$ and $U$-irreducible representations of Lie groups in the chain

$$
\mathrm{SO}(3, \mathrm{R}) \subset \mathrm{G}_{2} \subset \mathrm{SO}(7, \mathrm{R}) \subset \mathrm{U}_{7} .
$$


Such a classification problem is to be solved for each star $\gamma$. Here we are not going to solve this problem, but we want to point out some specific features associated with the permutational structure of the orbital space $L_{0}(\gamma)$. According to Eq. (19), $L_{0}(\gamma)$ is just the linear extension of the star $\gamma$, i.e. of an orbit of the transitive representation $R^{Q: H}$ of the symmetry group $Q$ of the box confining electrons, with $H$ being the stability group of an arm of the star $\gamma$. In particular, for $N=2$ one has to deal with Mackey theorem for transitive representations, which can be looked at as the crystalline analogue of coupling of two angular momenta - the building block for all Racah recoupling schemes [1-3]. We thus arrive in a natural way to discussion of the problem of multiple coupling of transitive rather than irreducible representations. We express our hope that it can shed a new light to electron correlations in crystals.

The Wigner-Racah approach yields an important quantum number - the Young diagram $\Lambda$ given by Eq. (43). This diagram describes the statistic of the orbital part of an $N$-electron wave function, i.e. its properties under permutations of electrons. $A$ describes, in general, neither fermions nor bosons but some paraparticles (actually parafermions with $p=2$ ) corresponding to multidimensional irreducible representations of the Pauli group $\Sigma_{N}$ (Ohnuki and Kamefuchi [15]). Such a description is convenient in construction of multielectron states in a spirit of Weyl duality [14], but it introduces complications to methods of second quantization. It is, however, a natural result of factorization (21) combined with the Pauli principle.

Evidently, the free-electron model suffers from some crude assumptions. In particular, such a full and, moreover, systematic neglect of interactions between electrons is inadequate in the picture of shells. It is rather reasonable to admit that more than one shell is partially filled. Such a partial filling of rungs near the Fermi level is an analogy of mixed valence approach in the tight binding model. It provides still larger flexibility in construction of states with various paramagnetic properties, resulting from both spin and orbital effects.

Also the quantum number $\gamma$ of a star in the reciprocal space ceases to be exact in presence of interactions. Still, we believe that the assumption of a single star is a good starting point for searching of electron correlations in extended systems. In particular, it seems to be a reasonable assumption in the case of such systems at the mesoscopic scale, when quantum size effects become important.

\section{References}

[1] E.P. Wigner, Group Theory and Its Application to the Quantum Mechanics of Atomic Spectra, Academic Press, New York 1959.

[2] U. Fano, G. Racah, Irreducible Tensorial Sets, Academic Press, New York 1959.

[3] L.C. Biedenharn, J.D. Louck, The Racah-Wigner Algebra in Quanlum Theory, Encycl. Math. Vol. 9, Addison-Wesley, Reading, Massachusetts 1981.

[4] J. Biel, R. Chatterjee, T. Lulek, J. Chem Phys. 86, 4531 (1987).

[5] T. Lulek, Acta Phys. Pol. A75, 487 (1989).

[6] T. Lulek, M. Szopa, J. Phys. A 23, 677 (1990). 
[7] J. Kawacki, J. Kłos, T. Lulek, Acta Phys. Pol. A78, 659 (1990).

[8] T. Lulek, in Symmetry and Structural Properties of Condensed Matter, Eds. W. Florek, T. Lulek, M. Mucha, World Sci., Singapore 1991, p. 278.

[9] B. Lulek, T. Lulek, J. Biel, R. Chatterjee, Can. J. Phys. 63, 1065 (1985).

[10] B.G. Wybourne, Spectroscopic Properties of the Rare Earths, Wiley, New York 1965.

[11] W. Wanagas, Algebraic Methods of the Theory of Nucleus, Mintis, Vilnius 1971 (in Russian).

[12] J. Karwowski, in Ref. [8], p. 258.

[13] M. Kuźma, B. Lulek, T. Lulek, Acta Phys. Pol. A75, 633 (1989).

[14] H. Weyl, The Theory of Groups and Quantum Mechanics, Dover, New York 1950.

[15] Y. Ohnuki, S. Kamefuchi, Quantum Field Theory and Parastatistics, Springer-Verlag, Berlin 1982. 